\title{
Arquitectura de Big Data para la gestión de incendios forestales en la región de La Araucanía
}

\author{
Big Data Architecture for Forest Fire Management Support in the Region of Araucanía
}

Arquitetura de Big Data para gestão de incêndlios florestais na região de La Araucanía

Felipe Vásquez-Morales ${ }^{1}$

Ania Cravero-Leal ${ }^{2}$

Recibido: mayo de 2021

Aceptado: agosto de 2021

Para citar este artículo: Vásquez-Morales, F. y Cravero-Leal, A.(2021). Arquitectura de Big Data para la gestión de incendios forestales en la región de La Araucanía. Revista Científica, 42(3), 304-314. https://doi. org/10.14483/23448350.18349

\section{Resumen}

Los incendios forestales han sido un problema que ha ido en aumento en las últimas décadas. En los recientes años se ha estado utilizando tecnología de Big Data para el procesamiento de grandes volúmenes de datos provenientes de sensores, fotos, imágenes satelitales y datos de valor de la experiencia en terreno. En Chile, no existen sistemas de Big Data para apoyar la gestión en incendios forestales. En este trabajo se busca proponer una arquitectura de Big Data para la gestión del volumen de datos provistos por imágenes satelitales, y que apoye la gestión de incendios en Chile. Esta arquitectura fue probada por medio de un prototipo implementado con herramientas de Cloud Computing, procesando imágenes satelitales, enfocado en el análisis de quemas controladas en la región de La Araucanía. Los resultados muestran que las imágenes resultantes son valiosas para la toma de decisiones en la gestión de quemas dentro de la región. Si bien existe mucho que mejorar, los resultados son alentadores de cara al valor que generan las imágenes resultantes, y a la mejora de este prototipo y de la propia arquitectura.

Palabras clave: arquitectura; computación en la nube; big data; imágenes satelitales; incendios forestales; prototipo.

\begin{abstract}
Wildfires have been a growing problem in the last decades. In recent years, Big Data technology has been used to process large volumes of data from sensors, photos, satellite and images, as well as valuable data from field experience. In Chile, there are no Big Data systems to support forest fire management. This work aims to propose a Big Data architecture for managing the volume of data provided by satellite images and supporting fire management
\end{abstract}

1. Universidad de La Frontera, Temuco, Chile. felipe.vasquez@ufrontera.cl

2. Universidad de La Frontera, Temuco, Chile. ania.cravero@ufrontera.cl 
in Chile. This architecture was tested through a prototype implemented with Cloud Computing tools, which processes satellite images and is focused on the analysis of controlled burns in the region of La Araucanía. The results show that the resulting images are valuable for decision-making in the management of burns within the region. Although there is much to improve, the results are encouraging in terms of the value generated by the resulting images and the improvement of this prototype and the architecture itself.

Keywords: architecture; big data; cloud computing; forest fire; prototype; satellite images.

\section{Resumo}

Os incêndios florestais têm sido um problema crescente nas últimas décadas. Nos últimos anos, a tecnologia de Big Data tem sido usada para processar grandes volumes de dados de sensores, fotos, imagens de satélite e dados valiosos da experiência de campo. No Chile, não existem sistemas de Big Data para apoiar o manejo de incêndios florestais. Este trabalho busca propor uma arquitetura de Big Data para gerenciar o volume de dados fornecidos por imagens de satélite, e que apoie o manejo do fogo no Chile. Esta arquitetura foi testada através de um protótipo implementado com ferramentas de Cloud Computing, processando imagens de satélite, com foco na análise de queimadas controladas na região de La Araucanía. Os resultados mostram o quanto há valor nas imagens resultantes, voltadas para a tomada de decisão para o manejo das queimadas na região. Embora haja muito a melhorar, os resultados são animadores face ao valor gerado pelas imagens resultantes, e ao aperfeiçoamento deste protótipo e da própria arquitetura.

Palavras-chaves: arquitetura; big data; cloud computing; imagens de satélite; protótipo; queimada.

\section{Introducción}

Las áreas forestales tienden a ser entornos complejos que cubren un tercio de las zonas terrestres del planeta (Moayedi et al., 2020). Estos entornos se ven afectados por diversos factores ambientales y antrópicos, siendo el más peligroso y destructivo los incendios forestales (F. Wu, Lv y Zhang, 2019), debido a su velocidad de propagación. A nivel mundial han afectado aproximadamente a 67 millones de hectáreas entre 2003 y 2012 (Wu et al., 2020).

Es posible apreciar el impacto causado por un incendio forestal en función del daño causado al ecosistema (flora y fauna) y a todo tipo de organismos existentes dentro del perímetro afectado, además de que minimiza la capacidad regenerativa del propio bosque y la conservación del recurso hídrico (Wu, Lv y Zhang, 2019).

Los incendios forestales se clasifican en tres grandes áreas según su ubicación y tamaño. La primera, conocida como incendio subterráneo, en el que solo el humo es visible y no se aprecia ningún tipo de llama. Otra es el incendio en superficie, que implica humo y Ilamas visibles. En caso de no controlar este segundo caso se llega al tercer tipo, el incendio forestal de corona o copa, que es el más destacado y conocido por sus llamas altas y por abarcar grandes cantidades de bosque (Chowdary, Kumar y Singh, 2018). Por otro lado, dentro de la literatura, los diferentes tipos de incendios forestales pueden ser clasificados también en dos grupos, prescritos o controlados, y además los incendios sin control, que tienden a crecer indefinidamente (Certini, 2005).

Para actuar apropiadamente sobre un incendio forestal descontrolado, se debe ser consciente de la situación y conocer el mayor detalle posible con el fin de tomar decisiones (Zambrano et al., 2017).

A nivel nacional, la toma de decisiones y todas las actividades relacionadas con el combate de incendios forestales es realizado por la Corporación Nacional Forestal (CONAF), que Ileva a cabo estadísticas históricas de los incendios a nivel nacional. Los datos indican que la cantidad de incendios durante el último periodo estadístico (julio 2019 - junio 2020) ha aumentado un $55 \%$ respecto al primer registro estadístico que se posee, correspondiente al periodo de 19851986, alcanzando los 8127 incendios forestales (CONAF, 2020a). 
CONAF posee tres plataformas informáticas para el apoyo a la toma de decisiones. La primera plataforma es Kitral, utilizada desde el año 1996, la cual es un sistema de información geográfico (GIS) encargado de realizar los trabajos relacionados con la predicción del avance de los incendios, utilizando diferentes variables ambientales (Ritchie, 1999).

La segunda herramienta es un sistema de registros, conocido como Sistema de Asistencia a Quemas controladas (SAQ), que es un protocolo online que permite el registro de las quemas controladas a realizarse por agricultores en sus predios para la limpieza de rastrojos, la cual se utiliza también por parte de carabineros con el fin de realizar un seguimiento a la información registrada y evitar propagación de incendios (CONAF, 2020b).

Finalmente, fuera de los sistemas oficiales publicados, se encuentra el tercer sistema, que está asociado a denuncias. El sistema funciona a través de smartphones, permitiendo a los brigadistas en terreno utilizar las cámaras de sus teléfonos móviles en conjunto con una breve descripción, con el fin de informar acerca de potenciales focos de incendio en los diferentes sectores que recorren.

En la actualidad todos estos sistemas trabajan de manera independiente, siendo estos dos últimos procesados manualmente (cada solicitud o denuncia debe comprobarse de manera manual por un trabajador de CONAF).

Por otro lado, las tecnologías de Big Data se han estado utilizando en diversos países para el apoyo a la toma de decisiones en incendios forestales. Esto debido a la capacidad de procesar un volumen datos estructurados y no estructurados, para luego obtener datos de valor. En Sayad, Mousannif y Al Moatassime (2019) se utilizó un sistema de Big Data, que incluye la teledetección y minería de datos (redes neuronales artificiales y SVM) para procesar los datos recolectados de imágenes satelitales en grandes áreas y extraer ideas de ellos para predecir la ocurrencia de incendios forestales. Los experimentos se Ilevaron a cabo utilizando la plataforma Big Data Databricks. Los resultados experimentales ofrecieron una alta precisión de predicción (98,32\%).

En Bielski et al. (2017) utilizaron tecnología Big Data para manejar incendios en un ámbito más general, desde la educación de la población, pasando por brindar sistemas de monitoreo, hasta distribuir información de manera oportuna cuando ocurre un incendio. Los autores utilizan información de múltiples fuentes, obteniendo datos climatológicos y de las redes sociales. Una vez procesados los datos con los que se detecta un incendio, el sistema genera información para la toma de decisiones. El sistema brinda apoyo con información relevante a la ciudadanía o los habitantes del sector afectado para evitar pérdidas humanas. El mayor impacto es garantizar la protección de la vida humana.

CONAF no administra información diaria acerca de los diferentes factores que pueden influir en la propagación del incendio, tales como la temperatura, la humedad relativa del aire, la presión atmosférica, la cantidad de combustible en los alrededores, las condiciones geográficas del lugar, entre otros varios factores que deben ser considerados. En este sentido, un sistema de Big Data permitiría analizar todos estos datos según las necesidades de los tomadores de decisiones, las necesidades de los brigadistas y de las personas que viven en las cercanías.

En este trabajo se propone una arquitectura de Big Data para procesar datos de imágenes satelitales a través de herramientas Cloud. Esta arquitectura será validada a través de un prototipo desarrollado con base en un caso de uso, asociado a la gestión de quemas controladas en los predios frente a la limpieza de rastrojos asociados al área agrícola.

De cara a la comunidad científica, el aporte principal se asocia con la poca existencia de arquitecturas de Big Data (sección 2) encontradas en la literatura, y a la inexistencia de sistemas de Big Data asociados a computación en la nube en gestión de incendios forestales. 
El trabajo está compuesto por las siguientes secciones: en primer lugar, la sección de trabajos relacionados, continuando con la propuesta de arquitectura de Big Data, los resultados del trabajo realizado, los cuales incluyen la implementación del prototipo del caso de uso y sus productos resultantes, y finalmente las conclusiones.

\section{Trabajos relacionados}

Con el fin encontrar trabajos relacionados con este tema, se llevó a cabo una búsqueda sistemática de estudios, utilizando la metodología de mapeo sistemático, la cual se encarga de definir procesos que permiten reconocer y categorizar los resultados que han sido publicados en un área determinada (Petersen et al., 2008).

Esta búsqueda fue realizada a través de los motores de bases de datos Scopus y Google Scholar, donde se encontraron solo diez trabajos que explican el uso de herramientas de Big Data para el apoyo en incendios forestales (Gonçalves et al., 2019; Lin et al., 2018; Bielski et al., 2017; Athanasis et al., 2018; Tavra, Racetin y Peroš, 2019; Saadat y Husen, 2018; Athanasis et al., 2019; Sayad, Mousannif y Al Moatassime, 2019; Athanasis, Themistocleous y Kalabokidis, 2017; Zou et al., 2019). Por otro lado, solo dos trabajos explican la arquitectura de Big Data utilizada, lo que dificulta el análisis de las herramientas usadas. Ninguno de los trabajos plantea herramientas de computación en la nube, lo que indica que este tipo de sistemas aún se encuentra en desarrollo.

Una de las arquitecturas definidas es la propuesta por Athanasis et al. (2018). Si bien se relaciona con el área de incendios forestales, se enfoca en un problema y una solución totalmente diferentes. Su principal enfoque es el uso de una red social (Twitter) y su potencial de mejorar herramientas de apoyo con los datos masivos existentes dentro de la plataforma. En la Figura 1 se presenta la arquitectura detallada por Athanasis (2018).

Por otro lado, Zou et al. (2019) realizan una encuesta en la que se propone una arquitectura de Big Data general para el apoyo a la toma de decisiones en contexto forestales (Figura 2). Los autores mencionan distintas fuentes de datos que deben ser utilizados, internet de las cosas (IoT), redes sociales (RRSS), sensores remotos (RS), datos obtenidos desde encuestas (Surveying Data) y data asociada a la localización. Para el análisis de los datos utilizan Data Mining y Machine Learning, además de un visualizador que permite obtener información de incendios forestales.



Figura 1. Propuesta de arquitectura de Big Data para la administración de incendios forestales, según Athanasis et al. (2018)

Ambas arquitecturas sirven de base para diseñar una arquitectura de Big Data que se adapte a las necesidades en Chile. 


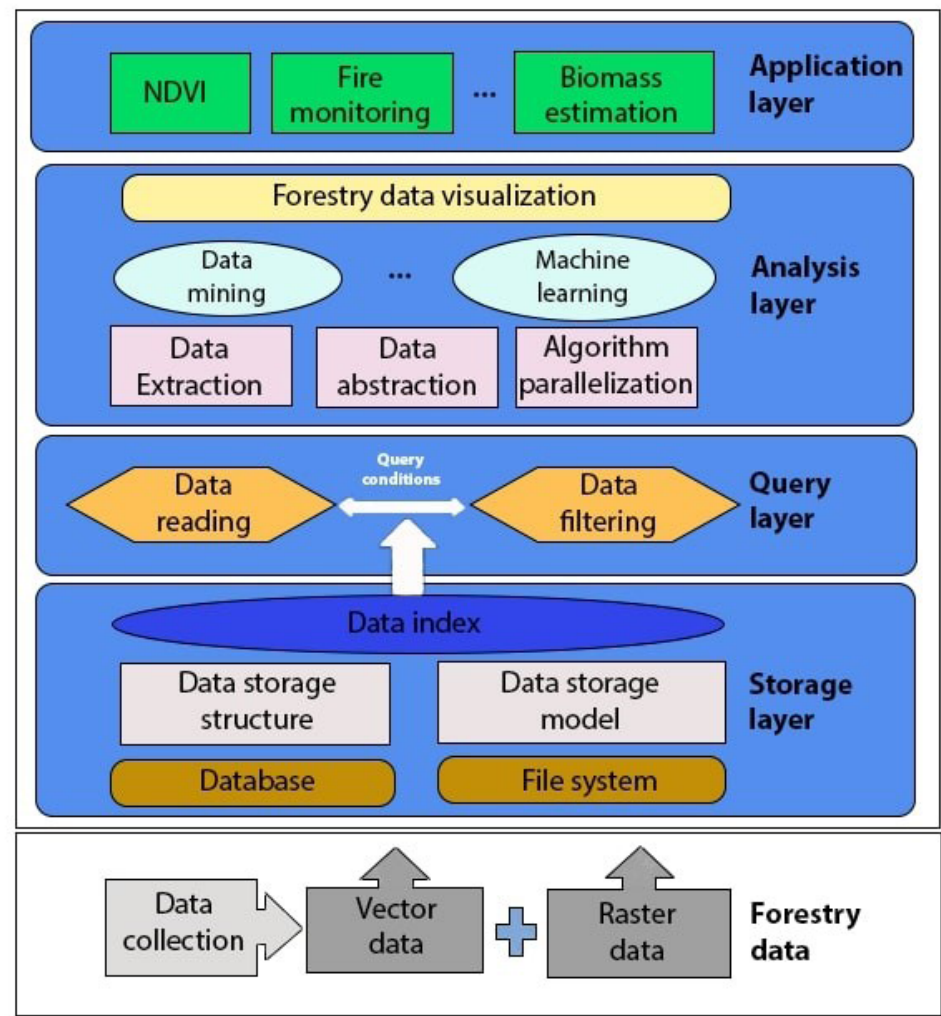

Figura 2. Generalización de arquitecturas para sistemas de Big Data asociados a contextos forestales según Zou et al., 2019

\section{Propuesta de arquitectura de Big Data}

Uno de los principales problemas relacionados con las arquitecturas encontradas en la sección anterior se relaciona con que ninguno de los escritos puede ser aplicado directamente en la región, debido a las diferencias de información disponible, así como de tecnologías y recursos.

La propuesta de arquitectura de Big Data que se diseñó (ver Figura 3) fue con base en la información disponible en la región de La Araucanía. Los datos que es posible extraer son aquellos provenientes de las herramientas de CONAF, y de sensores utilizados por Inia Carillanca; sin embargo, debido a que muchos de los datos son almacenados en bruto, sin validar, se debió utilizar datos provenientes de imágenes satelitales disponibles diariamente.

De acuerdo con lo discutido con miembros del laboratorio de teledetección satelital de la
Universidad de La Frontera, las imágenes satelitales pueden cubrir el espectro de información sobre temperatura y humedad.

El problema de esta información es que no permite obtener datos en tiempo real, sino que es información histórica. Sin embargo, existe otro conjunto de datos, obtenido a través del European Center for Medium-Range Weather Forecast (ECMWF), los cuales mediante el uso de herramientas de predicción y modelamiento matemático generan un modelo de clima que abarca diferentes factores, incluyendo presión atmosférica, dirección del viento y temperatura. El modelo entrega información, al igual que otras imágenes satelitales, con pixeles asociados temperatura, presión atmosférica, velocidad del viento, humedad relativa del aire, pero todo con base en predicciones que no son $100 \%$ exactas (ECMWF, 2021).

Otra variable que se encuentra presente es el conocimiento de los diferentes expertos con 
experiencia en el combate de incendios, esta información es totalmente empírica; sin embargo, es posible digitalizarla a través de bases de datos estructuradas, definiendo la zona a ser considerada y la opinión del experto, con base en niveles de peligrosidad.

La arquitectura de la Figura 3 está basada en el caso de uso para el apoyo a la gestión de quemas realizadas por agricultores. Se ha seleccionado este caso de uso debido a que es uno de los motivos importantes que provoca incendios forestales en la región de La Araucanía. Por otro lado, es un caso en desarrollo por el laboratorio de teledetección de La Universidad de La Frontera (UFRO). Esto permite validar de manera rápida la arquitectura a través de un prototipo funcional.

Los datos utilizados en el prototipo son imágenes satelitales que proveen información topográfica, niveles fluviales, cantidad de combustible existente en el área, entre otros variados factores.

Toda la información disponible permite relacionar los sectores donde se notificaron quemas controladas a realizarse en el futuro con el clima, la cantidad de combustible, la humedad ambiental y las opiniones de los expertos en el área, con el fin de permitir o posponer las quemas en los sectores y así evitar potenciales focos de incendios que generen pérdidas millonarias.

La arquitectura se compone de cinco capas principales:

\section{Ingesta de datos}

Los datos en una primera etapa se encuentran separados, por una parte, los modelos matemáticos de predicción climatológica del ECMWF en conjunto con las imágenes satelitales de las diferentes fuentes existentes almacenadas en el propio servidor de AWS dentro de buckets públicos de S3; por otra parte, la información que se genera de forma local se divide en dos componentes: los puntos de referenciación geográfica, sobre los cuales los agricultores realizarán quemas dentro de sus respectivos predios, y la opinión de los expertos, la cual puede adaptarse a condiciones lógicas para ser integradas en el sistema posteriormente.

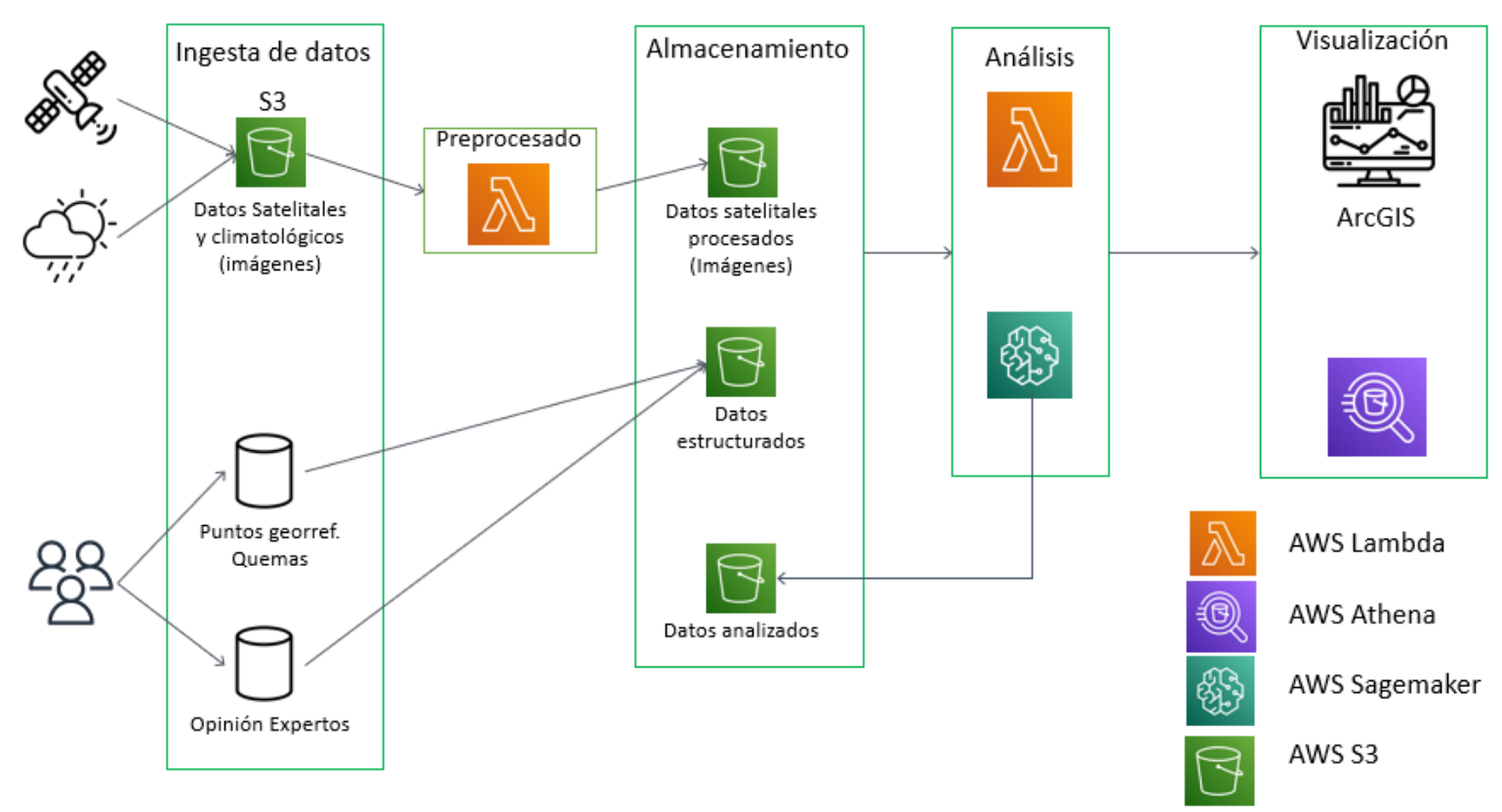

Figura 3. propuesta de arquitectura de Big Data para la administración de incendios forestales.

Fuente: elaboración propia 
Estas últimas pueden dar la sensación de ambigüedad, pero con base en los conocimientos de los expertos se puede ejemplificar que si el viento se encuentra apuntando hacia el este a más de 40 kilómetros por hora, tiende a ser un riesgo de incendio mayor que si apunta hacia al sur. Todas estas opiniones pueden ser interpretadas como factores lógicos para la toma de decisiones.

\section{Preprocesado}

Las imágenes satelitales son a grandes rasgos un conjunto de imágenes solapadas entre sí. En ese momento es cuando se requiere una etapa de preprocesado para separar las imágenes y obtener los valores y la información que estas traen incorporadas. Todo esto a través de procesamiento serverless utilizando scripts de AWS Lambda, los cuales permiten recibir, procesar y enviar la información generada adonde sea requerido.

\section{Almacenamiento}

Una vez procesados y ordenados los datos en su primera instancia, se procede a almacenarlos en diferentes buckets dentro de S3, con el fin de mantener el orden y evitar la mezcla de datos procesados y no procesados.

\section{Análisis}

Teniendo los datos almacenados y procesados lo suficiente para poder extraer valor de ellos, se procede a utilizar herramientas serverless como AWS Lambda, encargada de la generación de todas las imágenes resultantes, las cuales entregan toda la información acerca de las zonas con mayor peligro potencial de incendio. Además de Lambda, hace aparición AWS Sagemaker, herramienta serverless encargada de Machine Learning, permitiendo generar predicciones respecto al comportamiento de las variables con base en los resultados previos que haya tenido el mismo sistema.

\section{Visualización}

Dentro de la visualización, la principal herramienta a utilizar es de tipo GIS, esto es necesario dado que los resultados de los análisis asociados al área de Lambda terminan siendo imágenes georreferenciadas. Si bien AWS no cuenta con sistemas GIS, permite dentro de su Marketplace adquirir software de terceros. ArcGIS es una herramienta que permite visualizar imágenes en el formato TIFF, que es el asociado a componentes satelitales. Por otro lado, una vez vaya adquiriendo más información el sistema, se plantea utilizar AWS Athena para realizar consultas de información estructurada, principalmente asociada al área de los puntos de georreferenciación, o las diferentes opiniones de expertos almacenadas dentro del sistema.

Una ventaja de la arquitectura propuesta es el uso de una plataforma Cloud, ya que dispone de repositorios con imágenes satelitales y, por lo tanto, el procesamiento de los datos es más rápido. Por otro lado, las plataformas Cloud proveen espacio para manipular volumen de datos sin problemas.

En la Figura 4 se presenta el flujo de datos que fue implementado en el prototipo. Se utilizó la plataforma educativa de AWS.

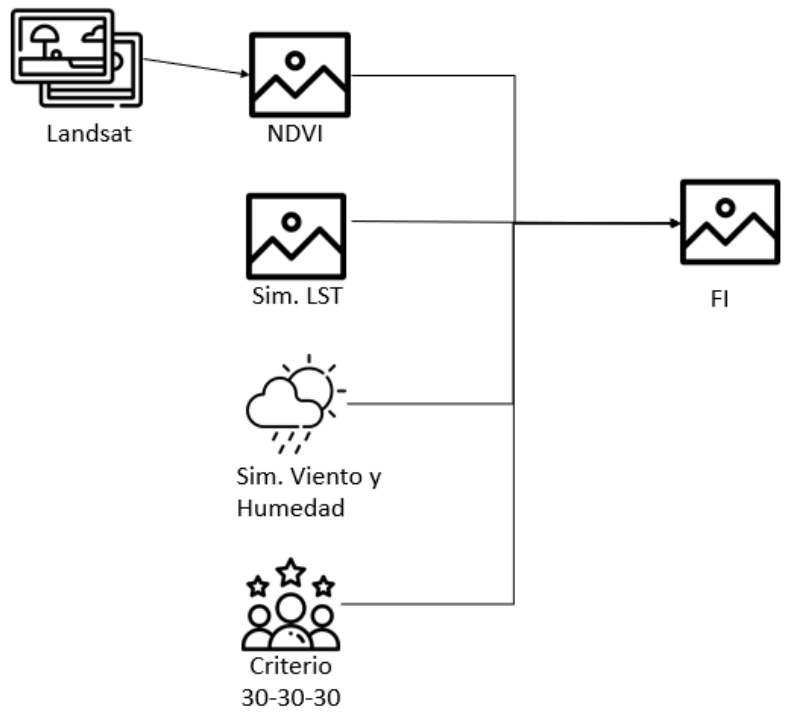

Figura 4. Representación visual del flujo de datos utilizados para la generación del FI en prototipo.

Fuente: elaboración propia 
Dentro de los materiales utilizados para el prototipo, se optó por utilizar imágenes satelitales provenientes del programa Landsat, para generar el índice de vegetación de diferencia normalizada (conocida por sus siglas en inglés como NDVI). Según el Earth Observation System, programa de la NASA para la observación terrestre, el NDVI es una medición del estado de salud de las plantas (y la existencia de las mismas), basado en cómo las plantas reflejan luz a diferentes frecuencias (EOS Data Analytics, s.f.). En este caso, el NDVI permite conocer la existencia o no de combustible en el sector estudiado, considerando combustible la vegetación en general. Para la obtención de esta se utilizan la banda de reflectancia en el espectro rojo (RED) y la banda de reflectancia en el espectro cerca del infrarrojo (conocido también como near-infrared spectrum o NIR). La Ecuación 1 demuestra cómo el cálculo es aplicado, todo considerando que se trabaja con matrices numéricas asociadas a las propias imágenes satelitales.

$$
N D V I=\frac{(N I R-R E D)}{(N I R+R E D)} \quad \text { Ecuación } 1
$$

Dentro de los componentes simulados se encuentra la temperatura superficial de la tierra, abreviada en su sigla LST, siendo esta diferente a la temperatura ambiental. Una de las formas de obtención de este dato es a través del Espectrorradiómetro de imágenes de media resolución (MODIS), el cual proporciona la temperatura diaria a nivel de superficie (NASA, 2020). Al igual que en el caso anterior, es una imagen que debe ser procesada para obtener su respectivo producto. Este componente será simulado debido a problemas de comunicación con los consultores asociados al laboratorio de teledetección.

Otros componentes simulados fueron los factores de velocidad del viento como la humedad relativa del aire, componentes que en un concepto funcional deben ser extraídos directamente desde el modelo de ECMWF, pero que en este caso por temas de tiempo fueron simulados de la forma más cercana a la realidad.

Finalmente, se utilizó un criterio de experto simulado para la determinación de los índices de peligro, extraído de la literatura. Se utilizó la conocida como "regla del 30-30-30" mencionada por Arana-Pulido et al. (2018), que según los consultores asociados al equipo es una de las reglas aplicadas dentro de la propia CONAF. Esta regla hace referencia a que existe una alta posibilidad de propagación de un incendio si la humedad está bajo el $30 \%$, la temperatura alcanza $30^{\circ} \mathrm{C}$ o más, y el viento tiene una velocidad de $30 \mathrm{~km} / \mathrm{h}$ (Arana-Pulido et al., 2018).

Con base en lo anterior se busca generar una nueva imagen resultante con todas las variables mencionadas, comprobando el cumplimiento del criterio 30-30-30 y un NDVI sobre 0.2 (lo cual es considerado un índice de vegetación alto) en cada pixel de la imagen resultante, sumando un 1 al valor del pixel, por cada criterio cumplido, pudiendo variar desde 0 (nula posibilidad de propagación de incendio) hasta 4 (propagación de incendio inminente).

El prototipo permite visualizar imágenes con zonas marcadas, que son las que están en peligro de un incendio forestal.

El prototipo soluciona el problema solo para un caso de uso. Como trabajo futuro se espera actualizar la arquitectura para incorporar datos y procesos para casos como prevención de incendios por zonas riesgosas, diseño de rutas en caso de incendios, administración de incendios con datos en tiempo real.

\section{Resultados}

La propuesta de arquitectura de Big Data permite indagar todas las posibilidades de la incorporación de datos provenientes de distintas fuentes de datos como sensores, imágenes satelitales, datos de expertos, datos provenientes de CONAF, datos provenientes de INIA. En esta primera etapa de desarrollo, se obtuvo una arquitectura para el 
caso de quemas realizadas por granjeros y agricultores, ya que es un factor importante de incendios en la región de La Araucanía. Por otro lado, aumenta su relevancia al no existir otras arquitecturas de Big Data asociadas a tecnologías Cloud en el área, lo cual facilita y soluciona componentes de Big Data como son el volumen y la velocidad.

Un subproducto de la propia arquitectura son los componentes generados desde la implementación del prototipo definido previamente. Este prototipo permite la generación de imágenes que contienen coordenadas, por lo cual se puede solapar con un mapa y a través de los procesos de coloración que entregan los propios sistemas GIS se puede apreciar información real. En la Figura 5 se puede apreciar cómo en la simulación generada un sector cercano a Vilcún contiene una peligrosidad de 4 respecto a incendios forestales (representada en rojo), por lo cual, al realizar una quema en ese sector, es prácticamente inminente. Esta imagen fue generada simulando un entorno relativamente común en épocas de verano, temperaturas entre 25 y $45{ }^{\circ} \mathrm{C}$, velocidad del viento entre 20 y $60 \mathrm{~km} / \mathrm{h}$ y una humedad relativa entre 10 y $40 \%$. Con base en esto, es normal que gran parte de la imagen se aprecie anaranjada. Mientras que, con base en la misma lógica, en la Figura 6 vemos la imagen dentro de un sistema GIS, con puntos georreferenciados superpuestos, los cuales simulan las referencias asociadas a puntos de quemas controladas de rastrojos, siendo en este ejemplo no factibles, dado el rango de color encontrado en el área donde el punto está situado.

Por otro lado, en la Figura 5 se puede apreciar que los resultados obtenidos pueden generar información real, lo cual, bajo condiciones de valores no simulados, puede ayudar a la toma de decisiones con respecto a la gestión de las quemas realizadas en la región; en la misma figura se aprecia que en las inmediaciones de Vilcún se deben evitar las quemas durante el día que fue adquirida la información.

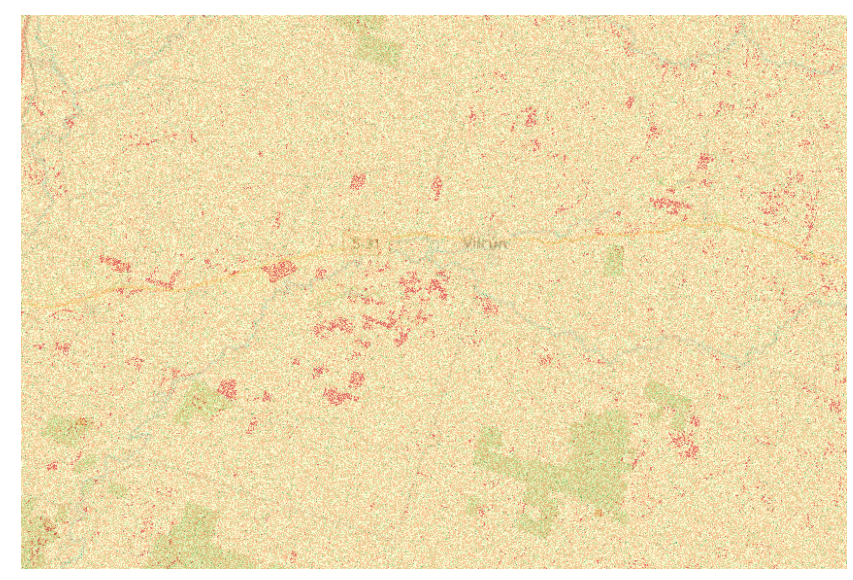

Figura 5. Imagen de elaboración propia coloreada y georreferenciada en el sector de Vilcún a través de QGIS.

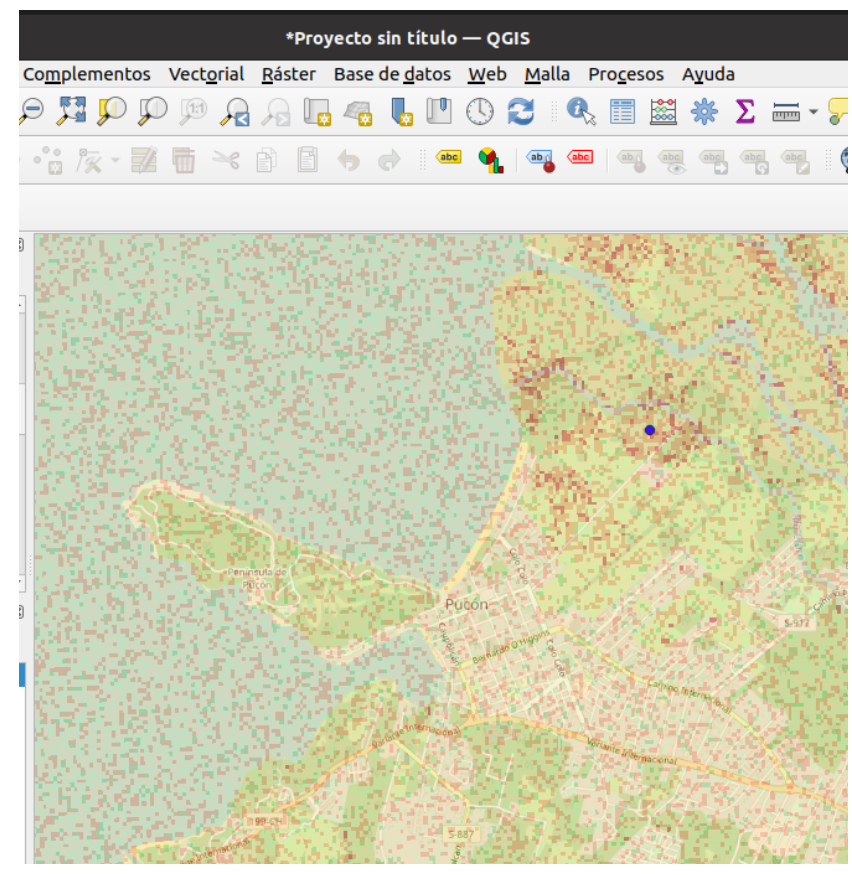

Figura 6. Imagen georreferenciada en el sector de Pucón con simulación de puntos notificados de quemas, utilizando QGIS.

\section{Conclusiones}

El trabajo presenta una propuesta de arquitectura de Big Data para la administración de incendios forestales en Chile. Para validar la arquitectura se ha implementado un prototipo que considera el caso de uso de incendios provocados por quemas de rastrojo en la región de La Araucanía. La 
arquitectura se diseñó considerando los tipos de datos disponibles en la región, ya que varias bases de daos o registros son validados a mano, o no son validados.

La arquitectura propuesta se compone de cinco capas que son implementadas utilizando herramientas disponibles en AWS Educate. Una de las ventajas es el rápido procesamiento de imágenes satelitales, debido a que AWS dispone de un repositorio de estas. Otra ventaja es el procesamiento de un volumen de datos estructurados y no estructurados, debido a que AWS dispone de espacio y herramientas preparadas para ello.

Se desarrolló un prototipo para el caso de uso de quemas de rastrojos, con el fin de probar la arquitectura. Los resultados muestran que es posible ampliar la arquitectura con otros datos provenientes de distintas fuentes.

Existe mucho potencial trabajo a futuro en el área, como inclusión de más imágenes satelitales para mejorar la precisión de las predicciones, especificar el cálculo de los productos en los componentes simulados y la obtención de estos, la propia mejora de la visualización dentro de la plataforma AWS, añadir técnicas de Machine Learning para un apoyo a la toma de decisiones más activo y no totalmente dependiente de quien aprecie los resultados generados. Además, este prototipo representa solo un caso de uso de la arquitectura, pudiendo en el futuro ser implementada de forma completa, comprobar su funcionamiento e ir añadiendo nuevos factores, asociados por ejemplo con el registro de incendios previos, la información de redes sociales, o adaptar el sistema para que no sea solo de prevención, sino que pueda adquirir tintes predictivos a través de las herramientas de Machine Learning que se pretenden añadir a futuro.

\section{Agradecimientos}

Este trabajo ha sido financiado por la Dirección de Investigación de la Universidad de La Frontera, Proyecto DI20-0014.

\section{Referencias}

Arana-Pulido, V., Cabrera-Almeida, F., Perez-Mato, J., Dorta-Naranjo, B. P., Hernandez-Rodriguez, S., Jimenez-Yguacel, E. (2018). Challenges of an autonomous wildfire geolocation system based on synthetic vision technology. Sensors, 18(11). https://doi.org/10.3390/s18113631

Athanasis, N., Themistocleous, M., Kalabokidis, K. (2017). Wildfire prevention in the era of big data. Lecture Notes in Business Information Processing, 299, 111-118. https://doi. org/10.1007/978-3-319-65930-5 9

Athanasis, N., Themistocleous, M., Kalabokidis, K., Chatzitheodorou, C. (2019). Big data analysis in uav surveillance for wildfire prevention and management. Lecture Notes in Business Information Processing, 341, 47-58. https://doi. org/10.1007/978-3-030-11395-7 5

Athanasis, N., Themistocleous, M., Kalabokidis, K., Papakonstantinou, A., Soulakellis, N., Palaiologou, P. (2018). The emergence of social media for natural disasters management: A big data perspective. International Archives of the Photogrammetry, Remote Sensing and Spatial Information Sciences - ISPRS Archives, 42(3W4), 75-82. https://doi.org/10.5194/ isprs-archives-XLII-3-W4-75-2018

Bielski, C. et al. (2017). Coupling early warning services, crowdsourcing, and modelling for improved decision support and wildfire emergency management. IEEE International Confernce on Big Data, 3705-3712. https://doi.org/10.1109/ BigData.2017.8258367

Certini, G. (2005). Effects of fire on properties of forest soils: A review. Oecologia, 143, 1-10. https://doi. org/10.1007/s00442-004-1788-8

Chowdary, V., Kumar Gupta, M., Singh, R. (2018). A Review on forest fire detection techniques: A decadal perspective. International Journal of Engineering \& Technology, 7(3.12). https://doi.org/10.14419/ijet. v7i3.12.17876

CONAF. (2020a). Incendios Forestales. Estadísticas Históricas. https://www.conaf.cl/incendios-forestales/ incendios-forestales-en-chile/estadisticas-historicas/ 
CONAF. (2020b). Sistema de asistencia a quemas controladas. https://saq.conaf.cl/login/index.php

ECMWF. (2021). Forecast charts and data. https://www. ecmwf.int/en/forecasts/

EOS Data Analytics. (s.f.). Normalized Difference Vegetation Index (NDVI). https://eos.com/ make-an-analysis/ndvi/

Gonçalves, D., Lima, D., Moura, J. M., Ferreira, L. (2019). "Portugal without fires", a data visualization system to help analyze forest fire data in Portugal. Lecture Notes of the Institute for Computer Sciences, Social Informatics and Telecommunications Engineering, 265, 520-525. https://doi. org/10.1007/978-3-030-06134-0_55

Lin, H., Liu, X., Wang, X., Liu, Y. (2018). A fuzzy inference and big data analysis algorithm for the prediction of forest fire based on rechargeable wireless sensor networks. Sustainable Computing: Informatics and Systems, 18, 101-111. https://doi.org/10.1016/j.suscom.2017.05.004

Moayedi, H., Mehrabi, M., Bui, D. T., Pradhan, B., Foong, L. K. (2020). Fuzzy-metaheuristic ensembles for spatial assessment of forest fire susceptibility. Journal of Environmental Management, 260. https://doi.org/10.1016/j.jenvman.2019.109867

NASA. (2020). MODIS Web. https://modis.gsfc.nasa. gov/data/dataprod/mod11.php

Petersen, K., Feldt, R., Mujtaba, S., Mattsson, M. (2008). Systematic mapping studies in software engineering. International Conference on Evaluation and Assessment in Software Engineering (EASE), 1-10. https://doi.org/10.14236/ewic/EASE2008.8

Ritchie, M. (1999). A compendium of forest growth and yield simulators for the Pacific coast states. Oxford University, $X X X, 149-155$

Saadat, N., Husen, M. N. (2018). An application framework for forest fire and haze detection with data acquisition using unmanned aerial vehicle. IMCOM '18: Proceedings of the 12th International Conference on Ubiquitous Information Management and Communication. https://doi. org/10.1145/3164541.3164624

Sayad, Y. O., Mousannif, H., Al Moatassime, H. (2019). Predictive modeling of wildfires: A new dataset and machine learning approach. Fire Safety Journal, 104, 130-146. https://doi.org/10.1016/j. firesaf.2019.01.006

Tavra, M., Racetin, I., Peroš, J. (2019). Combining social media and authoritative data for crisis mapping: A case study of a wildfire reaching Croatian city of split. International Archives of the Photogrammetry, Remote Sensing and Spatial Information Sciences, 42(3/W8), 415-420. https://doi.org/10.5194/ isprs-archives-XLII-3-W8-415-2019

Wu, F., Lv, X., Zhang, H. (2019). Design and development of forest fire monitoring terminal. 2018 International Conference on Sensor Networks and Signal Processing (SNSP), 40-44. https://doi.org/10.1109/ SNSP.2018.00017

Wu, Z., He, H. S., Keane, R. E., Zhu, Z., Wang, Y., Shan, Y. (2020). Current and future patterns of forest fire occurrence in China. International Journal of Wildland Fire, 29(2), 104-119. https://doi.org/10.1071/ WF19039

Zambrano, M., Esteve, M., Pérez, I., Carvajal, F., Zambrano, A. M. (2017). Situation awareness in the large forest fires response: A solution based on wireless mesh networks. 2017 IEEE 9th Latin-American Conference on Communications, 1-6. https:// doi.org/10.1109/LATINCOM.2017.8240147

Zou, W., Jing, W., Chen, G., Lu, Y., Song, H. (2019). A Survey of big data analytics for smart forestry. IEEE Access, 7, 46621-46636. https://doi.org/10.1109/ ACCESS.2019.2907999 